\title{
The Impact of High-Risk HPV Genotypes Other Than HPV 16/18 on the Natural Course of Abnormal Cervical Cytology: A Korean HPV Cohort Study
}

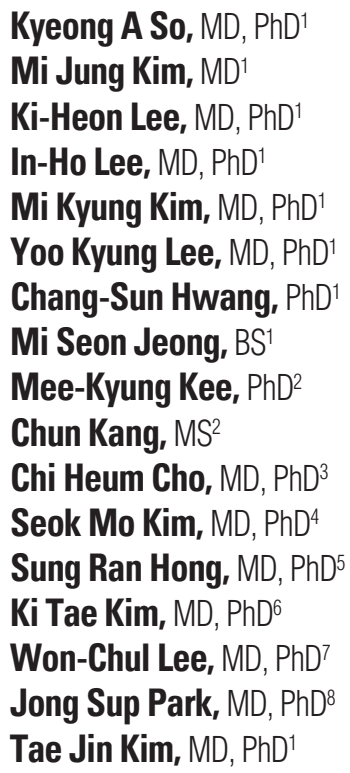

*A list author's affiliations appears at the end of the paper.

Correspondence: Tae Jin Kim, MD, PhD Department of Obstetrics and Gynecology, Cheil General Hospital and Women's Healthcare Center, Dankook University College of Medicine, 17 Seoae-ro 1-gil, Jung-gu, Seoul 04619, Korea

Tel: 82-2-2000-7577

Fax: 82-2-2000-7477

E-mail: kimonc111@naver.com

Co-Correspondence: Jong Sup Park, MD, PhD Department of Obstetrics and Gynecology, Seoul St. Mary's Hospital, College of Medicine, The Catholic University of Korea,

222 Banpo-daero, Seocho-gu, Seoul 06591, Korea Tel: 82-2-2258-7592

Fax: 82-2-2258-2724

E-mail: jspark@catholic.ac.kr

Received January 7, 2016

Accepted February 29, 2016

Published Online March 9, 2016

${ }^{*}$ Kyeong A So and Mi Jung Kim contributed equally to this work.

\section{Purpose}

The purpose of this study is to evaluate the impact of high-risk human papillomaviruses (HPVs) other than HPV 16/18 on the natural course of atypical squamous cells of undetermined significance (ASC-US) or low-grade squamous intraepithelial lesion (LSIL).

\section{Materials and Methods}

The study population was derived from the Korean HPV cohort (2010-2014). Women aged 20 to 60 who satisfied the criteria of having both HPV infection and abnormal cervical cytology of either ASC-US or LSIL were recruited from five institutions nationwide. Enrolled patients underwent cervical cytology and HPV DNA testing every 6 months.

Results

A total of 1,158 patients were enrolled. The 10 most common HPV types were HPV $16(12.3 \%), 58(10.0 \%), 56$ (8.8\%), 53 (8.4\%), 52 (7.7\%), 39 (6.2\%), 18 (6.0\%), 51 (5.7\%), 68 (5.1\%), and 66 (4.6\%). Among these patients, 636 women were positive for high-risk HPVs other than HPV 16 or 18, and 429 women were followed for more than 6 months. Cytology evaluations showed progression in $15.3 \%$ of women, no change in $22.6 \%$, and regression in $62.1 \%$ of women at 12 months. In cases of HPV 58 single infection, a more highly significant progression rate, compared to other high-risk types, was observed at 6 months (relative risk [RR], 3.3; 95\% confidence interval [Cl], 2.04 to 5.30; $p<0.001$ ) and 12 months (RR, 5.03; 95\% Cl, 2.56 to 9.91; $p<0.001$ ).

\section{Conclusion}

HPV genotypes numbered in the 50s were frequent in Korean women with ASC-US and LSIL. HPV 58 was the second most common type, with a high progression rate of cervical cytology. 


\section{Introduction}

More than 100 human papillomaviruses (HPVs) have been identified, among which approximately 20 types are known as high-risk or oncogenic types [1]. High-risk HPVs are the most important contributors to the development of cervical precancerous lesions and invasive carcinomas. The prevalence of cervical cancer varies remarkably in different countries; this is mainly related to the availability of cervical cancer screening programs [2]. In Korea, despite a decrease in cervical cancer incidence from 1999 to 2012 because of an organized screening program, it is still predicted to be the seventh most common female cancer with 3,100 new cases and the third most common cancer in women aged 15 to 34 in 2015 [3-5].

Persistent infection with high-risk HPVs is a primary risk factor for cervical carcinogenesis. Among the high-risk HPVs, HPV 16 and HPV 18 are the two most carcinogenic genotypes and current prophylactic vaccines against HPV $16 / 18$ may prevent up to $70 \%$ of cervical cancers [6]. Highrisk HPVs other than HPV 16/18 cause the remaining 25\%$35 \%$ of cervical cancers [7]. Globally, the most common genotypes other than HPV 16/18, in descending order of frequency are: HPV 45, 31, 33, 52, 58, and 35 [8]. However, there is considerable geographical variation in the prevalence of these HPV types. The prevalence of other high-risk HPV types is higher in Asia, Africa, and South/Central America (30\%-35\%) than in Europe, Oceania, and North America (23\%-26\%) [7]. Clarification of the contributions of individual genotypes causing cervical carcinogenesis will be important in order to provide an estimate of the potential impact of prophylactic vaccines and cancer screening programs.

This is a prospective study in a Korean HPV cohort [9]. The aims of this study were first to evaluate the distributional proportions along the full spectrum of high-risk HPVs in a Korean HPV cohort, and second, to determine the natural progression of atypical squamous cells of unknown significance (ASC-US) and low-grade squamous intraepithelial lesion (LSIL) with high-risk HPV infections other than HPV $16 / 18$.

\section{Materials and Methods}

A Korean HPV cohort study was conducted between April 2010 and October 2014. The HPV cohort recruited women aged 20 to 60 who satisfied both criteria of HPV positivity and abnormal cervical cytology of either ASC-US or LSIL from five institutions nationwide. Exclusion criteria included any of the following: previous hysterectomy, history of cervical intraepithelial neoplasia (CIN) treated within 6 months, pregnancy, coexisting malignancies, and psychological diseases currently under treatment. The study was approved by the institutional review board and informed consent was obtained from each participant prior to the study.

Enrolled patients underwent cervical liquid-based cytology and HPV DNA testing every 6 months. Cytological progression was defined as the change to atypical squamous cells-cannot exclude high-grade squamous intraepithelial lesion (HSIL), HSIL, and LSIL from ASC-US, or as the change to HSIL from LSIL. Regression was defined as the change to normal cytology or ASC-US from LSIL, or to normal cytology from ASC-US. The others were categorized as no change. Colposcopy-directed biopsies were performed in patients with cytologic results indicative of conditions worse than HSIL. Patients diagnosed as having a lesion classified as greater than CIN 2 by colposcopy-directed biopsy were terminated in this study. Cervical samples were collected using a Cervex-Brush (Rovers Medical Devices, Oss, Netherlands) from cervical os and SurePath liquid-based cytology (TriPath Imaging, Inc., Burlington, NC) was used during the followup examinations. Cheil General Hospital served as a central laboratory for cytological diagnoses according to the 2001 Bethesda reporting system [10], and HPV genotyping testing during the follow-up examinations. HPV genotyping was performed using a DNA microarray technique with a Cheil HPV DNA Chip Kit (Cheil General Hospital, Seoul, Korea), based on a polymerase chain reaction method. Briefly, postamplification SYBR Green reverse transcription quantitative polymerase chain reaction products were analyzed by $\mathrm{Ct}$ value and hybridized with probes for HPVs. Samples were analyzed for the presence of 19 high-risk HPV types $(16,18$, $31,33,35,39,45,51,52,53,56,58,59,66,67,68 \mathrm{a}, 68 \mathrm{~b}, 69$, and $82)$ and 17 low-risk HPV types (6, 11, 30, 32, 40, 42, 43, 44, 54, $55,62,70,72,81,84,90$, and 91$)$.

\section{Statistical analysis}

Data were analyzed using SPSS ver. 17.0 (SPSS Inc., Chicago, IL). Categorical variables were indicated as numbers and percentages. To assess the risk of cytological progression associated with HPV genotypes, relative risk (RR) with $95 \%$ confidence intervals (CI) was estimated by chisquare test. All p-values of less than 0.05 were considered statistically significant. 


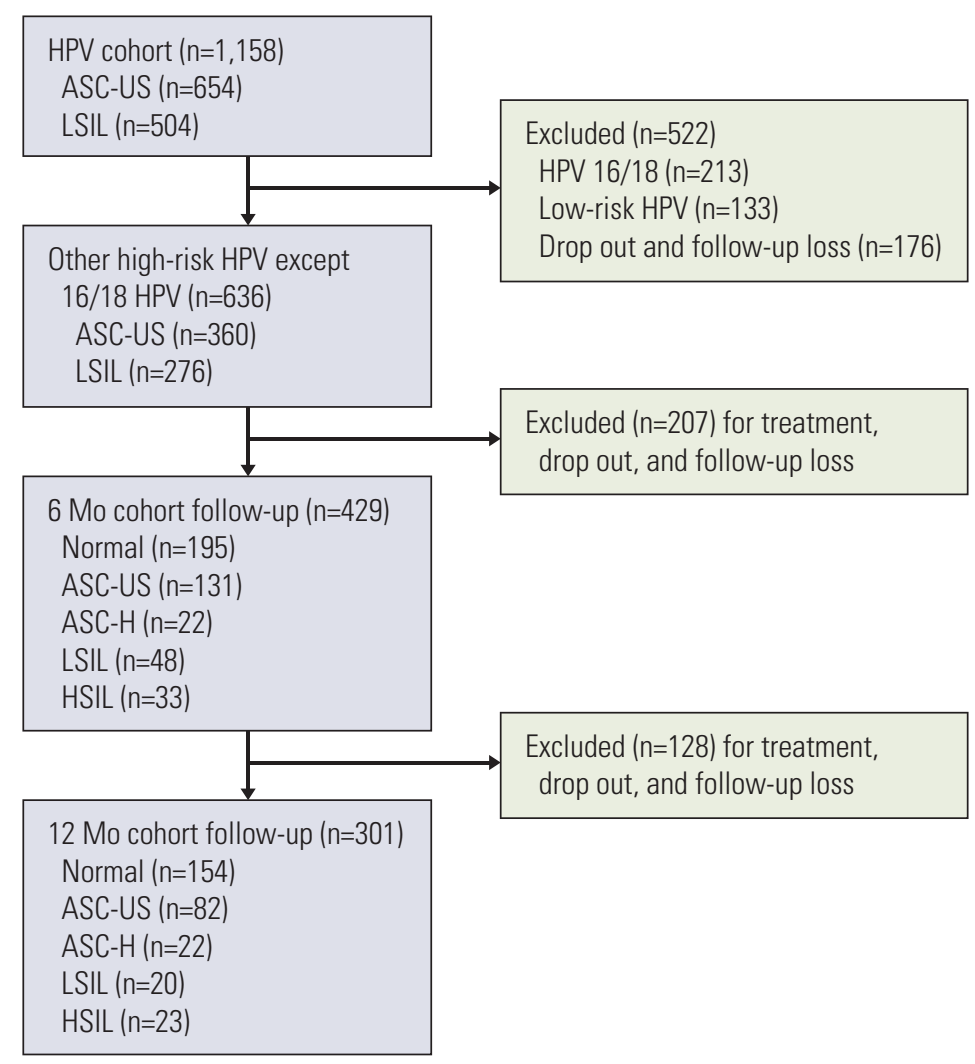

Fig. 1. Flow diagram of study selection. HPV, human papillomavirus; ASC-US, atypical squamous cells of undetermined significance; LSIL, low-grade squamous intraepithelial lesion; ASC-H, atypical squamous cells-cannot exclude high-grade squamous intraepithelial lesion; HSIL, high-grade squamous intraepithelial lesion.

\section{Results}

Among a total of 1,158 patients in the Korean HPV cohort study, 636 patients met the eligibility criteria of this study (Fig. 1). ASC-US and LSIL were observed in 360 patients $(56.6 \%)$ and 276 patients $(43.4 \%)$, respectively. With respect to follow-up, 429 patients $(67.5 \%)$ were followed up at 6 months and $301(47.3 \%)$ at 12 months. The prevalence of high-risk HPV infections at the time of enrollment is summarized in Table 1 . The most common type of high-risk HPV was type $16(12.3 \%)$ as a single infection $(n=85)$ and multiple infection ( $\mathrm{n}=58)$. In this cohort, HPV 58 was the second most common type, with 74 patients having a single infection and 42 patients having multiple infections. Only ten patients with HPV 58 were co-infected with HPV 16 or 18 . Among the ten most common high-risk HPVs, HPV 58, 56, 53, and 52 ranked as the top five. Cytological changes in patients with high-risk HPV infections other than HPV 16/18 during the follow-up periods are shown in Table 2. The rates of regression, no change, and progression of cervical cytology were $58.0 \%$, $26.1 \%$, and $15.9 \%$ at 6 months and $62.1 \%, 22.6 \%$, and $15.3 \%$ at 12 months; 113 of 360 cases of ASC-US (31.4\%) had regressed to normal cytology at 6 months; 136 of 276 cases of LSIL (49.3\%) had regressed to either ASC-US ( $n=54)$ or normal cytology $(n=82)$.

However, 147 of 301 (48.8\%) ASC-US/LSIL cases maintained abnormal cytology for 12 months. In the unchanged and progression groups, the persistent rates of high-risk HPV infections other than HPV 16/18 were markedly higher than in the regression group $(p<0.001)$. Among patients in the progression group, only three patients $(4.4 \%)$ and one patient $(2.2 \%)$ were HPV negative by HPV DNA test at 6 and 12 months, respectively. However, in the regression group, HPV infections had cleared in up to $62.6 \%$ of patients at the 12-month follow-up.

Analyses of high-risk HPV infections other than HPV $16 / 18$ showed persistence in $41.9 \%$, incidental infection in $24.6 \%$, and clearance in $33.5 \%$ of patients at 12 months; $36.5 \%$ of the persistent infections were identified as having been 
Table 1. Prevalence of HPVs among women with ASCUS or LSIL

\begin{tabular}{|c|c|c|c|}
\hline Category & Rank & HPV genotype & No. $(\%)(n=1,158)$ \\
\hline \multirow[t]{18}{*}{ High risk } & 1 & HPV 16 & $143(12.3)$ \\
\hline & 2 & HPV 58 & $116(10.0)$ \\
\hline & 3 & HPV 56 & $102(8.8)$ \\
\hline & 4 & HPV 53 & $97(8.4)$ \\
\hline & 5 & HPV 52 & $89(7.7)$ \\
\hline & 6 & HPV 39 & $72(6.2)$ \\
\hline & 7 & HPV 18 & $70(6.0)$ \\
\hline & 8 & HPV 51 & $66(5.7)$ \\
\hline & 9 & HPV 68 & $59(5.1)$ \\
\hline & 10 & HPV 66 & $53(4.6)$ \\
\hline & 11 & HPV 35 & $41(3.5)$ \\
\hline & 12 & HPV 33 & $37(3.2)$ \\
\hline & 13 & HPV 31 & $28(2.4)$ \\
\hline & 14 & HPV 59 & $24(2.1)$ \\
\hline & 15 & HPV 45 & $16(1.4)$ \\
\hline & 16 & HPV 67 & $6(0.5)$ \\
\hline & 17 & HPV 82 & $5(0.4)$ \\
\hline & 18 & HPV 69 & $4(0.3)$ \\
\hline \multirow[t]{15}{*}{ Low risk } & 1 & HPV 70 & $41(3.5)$ \\
\hline & 2 & HPV 40 & $33(2.8)$ \\
\hline & 3 & HPV 54 & $28(2.4)$ \\
\hline & 4 & HPV44 & $27(2.3)$ \\
\hline & 5 & HPV 62 & $23(2.0)$ \\
\hline & 6 & HPV 6 & $22(1.9)$ \\
\hline & 7 & HPV 81 & $20(1.7)$ \\
\hline & 8 & HPV 42 & $17(1.5)$ \\
\hline & 9 & HPV 30 & $16(1.4)$ \\
\hline & 10 & HPV 43 & $15(1.3)$ \\
\hline & 11 & HPV 11,84 & $14(1.2)$ \\
\hline & 12 & HPV 90 & $11(0.9)$ \\
\hline & 13 & HPV 91 & $10(0.9)$ \\
\hline & 14 & HPV 55 & $4(0.3)$ \\
\hline & 15 & HPV 32, 72 & $1(0.1)$ \\
\hline
\end{tabular}

Each type included single and multiple infection. HPV, human papillomavirus; ASC-US, atypical squamous cell uncertain significance; LSIL, low-grade squamous intraepithelial lesion.

caused by the same genotype on all HPV DNA tests. In addition, persistent infection with each HPV genotype showed a significant correlation with the prognosis of cervical cytology. The rates of persistent infection for other highrisk HPVs gradually increased from regression to progression in cytological prognosis $(\mathrm{p}<0.001)$.

With the exception of HPV 16/18, the HPV type-specific prognoses of cervical cytology are shown in Table 3 . The cytological progression rates of HPV 58, 56, 53, 52, 39, 51, 68, and 66 single infections were $30.3 \%, 2.9 \%, 7.7 \%, 13.1 \%$, $11.8 \%, 7.2 \%, 15.4 \%$, and $5.9 \%$, respectively, at 12 months. Except for HPV 58, low progression rates were observed for the other common HPV types (1.5\%-29.3\%) in both single and total infections. A relatively high progression rate (26.0\%-37.5\%) was observed for HPV 58 infection during the follow-up periods. The rate of cytological progression was $27.0 \%$ (27 out of 100) in the HPV 16 persistence group, and 9.8\% (4 out of 41) in the HPV 18 persistence group at 6 months of follow-up.

According to the genotype and follow-up duration, the RRs to progression of cytology are shown in Table 4. HPV 58 single infection with ASC-US/LSIL resulted in a significantly higher risk of progression, compared with other common high-risk HPVs at 6 and 12 months (RR, 3.30; 95\% CI, 2.04 to 5.30; $p<0.001$ and RR, 5.03; 95\% CI, 2.56 to 9.91; $\mathrm{p}<0.001$ ). Type 56,53 , and $52 \mathrm{HPVs}$, which initially ranked among the top five types, showed no significant RRs.

\section{Discussion}

In this study, HPV $16(12.3 \%)$ was clearly the predominant HPV type identified among the 1,158 women with ASC-US or LSIL, consistent with the universal trend among prevalent HPV types. However HPV 18 (6.0\%) was not frequent in our study. Instead of HPV 18, HPV $58(10.0 \%)$ was the second most common HPV type. This finding is consistent with those of other previous studies conducted in East Asia. The overall reported prevalence of HPV 58 was $11.4 \%$ in a study from Hong Kong [11]. In a study conducted in China, HPV 58 was more frequent than HPV 18 in precancerous lesions (19.1\% vs. 5.4\%, respectively) and the same infection rates for HPV 58 and HPV 18 were reported in cervical cancers $(9.4 \%$ each) [12]. HPV 58 accounts for a considerable disease burden in East Asia. Its prevalence in cervical cancer is higher in Asia (5.6\%) than in other parts of the world (3.3\%) [13]. In a large scale Korean study $(\mathrm{n}=1,650)$, HPV 58 was the second most common HPV in LSIL (8.1\%) and HSIL (15.7\%) [14]. HSIL has also shown a relatively high prevalence $(17.2 \%)$ of HPV 58 infection in East Asia [15].

The lifelong risk for HPV infection is greater than $70 \%$ in sexually active women [16]. Most HPV infections are spontaneously cleared within 1 to 2 years because of immunological mechanisms $[17,18]$. In a previous study evaluating HPV infections in adolescents, infections were spontaneously cleared by 12 months in over $50 \%$ of infections caused by high-risk HPV types and two-thirds of infections caused by low-risk HPV types [19]. In this study, the overall clearance rate of high-risk HPV types other than HPV 16/18 at 
Table 2. Prevalence of high-risk HPVs infection according to the prognosis of cervical cytology

\begin{tabular}{|c|c|c|c|c|c|}
\hline Category & Regression & No change & Progression & Total & p-value \\
\hline $6 \mathrm{Mo}$ & $249(58.0)$ & $112(26.1)$ & 68 (15.9) & $429(100)$ & $<0.001$ \\
\hline High-risk HPV (+) & $111(44.6)$ & $93(83.0)$ & 65 (95.6) & $269(62.7)$ & \\
\hline High-risk HPV (-) & $138(55.4)$ & $19(17.0)$ & $3(4.4)$ & $160(37.3)$ & \\
\hline $12 \mathrm{Mo}$ & $187(62.1)$ & 68 (22.6) & $46(15.3)$ & $301(100)$ & $<0.001$ \\
\hline High-risk HPV (+) & $70(37.4)$ & $49(72.1)$ & $45(97.8)$ & $164(54.5)$ & \\
\hline High-risk HPV (-) & $117(62.6)$ & $19(27.9)$ & $1(2.2)$ & $137(45.5)$ & \\
\hline
\end{tabular}

Values are presented as number $(\%)$. HPV, human papillomavirus.

Table 3. HPV type-specific prognosis for cervical cytology

\begin{tabular}{|c|c|c|c|c|c|}
\hline \multirow{2}{*}{ HPV genotype } & \multirow{2}{*}{ Prognosis of cytology } & \multicolumn{2}{|c|}{ Single infection } & \multicolumn{2}{|c|}{ Total infections } \\
\hline & & 6 Mo & $12 \mathrm{Mo}$ & 6 Mo & $12 \mathrm{Mo}$ \\
\hline \multirow[t]{3}{*}{ HPV 58} & Progression & $18(37.5)$ & $10(30.3)$ & $22(29.7)$ & $13(26.0)$ \\
\hline & No change & $10(20.8)$ & $6(18.2)$ & $18(24.3)$ & $12(24.0)$ \\
\hline & Regression & $20(41.7)$ & $17(51.5)$ & $34(46.0)$ & $25(50.0)$ \\
\hline \multirow[t]{3}{*}{ HPV 56} & Progression & $1(1.8)$ & $1(2.9)$ & $1(1.5)$ & $2(4.2)$ \\
\hline & No change & $11(20.0)$ & $2(5.9)$ & $22(33.3)$ & $7(14.6)$ \\
\hline & Regression & $43(78.2)$ & $31(91.2)$ & $43(65.2)$ & $39(81.2)$ \\
\hline \multirow[t]{3}{*}{ HPV 53} & Progression & $3(8.6)$ & $2(7.7)$ & $6(9.2)$ & $4(8.5)$ \\
\hline & No change & $6(17.1)$ & $4(15.4)$ & $13(20.0)$ & $10(21.3)$ \\
\hline & Regression & $26(74.3)$ & $20(76.9)$ & $46(70.8)$ & $33(70.2)$ \\
\hline \multirow[t]{3}{*}{ HPV 52} & Progression & $6(17.6)$ & $3(13.1)$ & $11(19.3)$ & $12(29.3)$ \\
\hline & No change & $11(32.4)$ & $5(21.7)$ & $18(31.6)$ & $9(21.9)$ \\
\hline & Regression & $17(50.0)$ & $15(65.2)$ & $28(49.1)$ & $20(48.8)$ \\
\hline \multirow[t]{3}{*}{ HPV 39} & Progression & $3(11.5)$ & $2(11.8)$ & $5(11.4)$ & $3(10.7)$ \\
\hline & No change & $7(26.9)$ & $5(29.4)$ & $13(29.5)$ & $10(35.7)$ \\
\hline & Regression & $16(61.6)$ & $10(58.8)$ & $26(59.1)$ & $15(53.6)$ \\
\hline \multirow[t]{3}{*}{ HPV 51} & Progression & $2(9.1)$ & $1(7.2)$ & $5(11.9)$ & $5(20.8)$ \\
\hline & No change & $5(22.7)$ & $3(21.4)$ & $14(33.3)$ & $5(20.8)$ \\
\hline & Regression & $15(68.2)$ & $10(71.4)$ & $23(54.8)$ & $14(58.4)$ \\
\hline \multirow[t]{3}{*}{ HPV 68} & Progression & $4(23.5)$ & $2(15.4)$ & $8(25.0)$ & $5(22.7)$ \\
\hline & No change & $4(23.5)$ & $3(23.1)$ & $9(28.1)$ & $5(22.7)$ \\
\hline & Regression & $9(53.0)$ & $8(61.5)$ & $15(46.9)$ & $12(54.6)$ \\
\hline \multirow[t]{3}{*}{ HPV 66} & Progression & $3(13.6)$ & $1(5.9)$ & 4 (11.4) & $2(8.0)$ \\
\hline & No change & $6(27.3)$ & $5(29.4)$ & $11(31.4)$ & $7(28.0)$ \\
\hline & Regression & $13(59.1)$ & $11(64.7)$ & $20(57.2)$ & $16(64.0)$ \\
\hline
\end{tabular}

Values are presented as number $(\%)$. HPV, human papillomavirus.

12 months was $33.5 \%$. HPV clearance was also higher in the cytological regression group than in the unchanged and progression groups. Overall, approximately $60 \%$ of patients showed regression on the subsequent follow-up Papanicolaou tests.

On the other hand, persistence of HPV infection was closely related to the prognosis of cervical cytology. Approximately $15 \%$ of patients showed cytological progression on every follow-up test. The progression group showed significantly higher rates of persistent infection with all other highrisk HPV types. Significant decreases in the rates of persistent infection were observed in the cytological regression, the unchanged, and progression groups, in order $(p<0.001)$. The results of this study with respect to cervical pathogenesis confirm that persistent high-risk HPV infection induces cervical carcinogenesis. In most cases, the persistence of high- 
Table 4. HPV type-specific relative risk to progression of cervical cytology

\begin{tabular}{|c|c|c|c|c|c|c|}
\hline \multirow{2}{*}{ HPV type } & \multicolumn{3}{|c|}{$6 \mathrm{Mo}$} & \multicolumn{3}{|c|}{$12 \mathrm{Mo}$} \\
\hline & No. & RR $(95 \%$ CI) & p-value & No. & RR $(95 \%$ CI) & p-value \\
\hline HPV 58 & $18 / 48$ & $3.3(2.04-5.30)$ & $<0.001$ & $10 / 24$ & $5.03(2.56-9.91)$ & $<0.001$ \\
\hline Others & $22 / 211$ & 1 & & $12 / 153$ & 1 & \\
\hline HPV 56 & $1 / 55$ & $0.10(0.14-0.72)$ & $<0.001$ & $1 / 14$ & $0.52(0.07-4.18)$ & 0.458 \\
\hline Others & $39 / 204$ & 1 & & $22 / 163$ & 1 & \\
\hline HPV 53 & $3 / 35$ & $0.51(0.17-1.60)$ & 0.170 & $2 / 15$ & $1.08(0.26-4.49)$ & 0.583 \\
\hline Others & $37 / 224$ & 1 & & $20 / 162$ & 1 & \\
\hline HPV 52 & $6 / 33$ & $1.22(0.54-2.76)$ & 0.401 & $2 / 18$ & $0.88(0.22-3.57)$ & 0.607 \\
\hline Others & $34 / 226$ & 1 & & $20 / 159$ & 1 & \\
\hline HPV 39 & $3 / 26$ & $0.71(0.23-2.27)$ & 0.404 & $2 / 10$ & $1.76(0.40-7.77)$ & 0.360 \\
\hline Others & $37 / 233$ & 1 & & $20 / 167$ & 1 & \\
\hline HPV 51 & $2 / 22$ & $0.55(0.13-2.25)$ & 0.306 & $1 / 12$ & $0.64(0.09-4.72)$ & 0.545 \\
\hline Others & $38 / 237$ & 1 & & $21 / 165$ & 1 & \\
\hline HPV 68 & $4 / 17$ & $1.69(0.58-4.90)$ & 0.257 & $2 / 10$ & $1.76(0.40-7.77)$ & 0.360 \\
\hline Others & $36 / 242$ & 1 & & $20 / 167$ & 1 & \\
\hline HPV 66 & $3 / 22$ & $0.86(0.27-2.80)$ & 0.549 & $1 / 28$ & $0.26(0.04-1.83)$ & 0.100 \\
\hline Others & $37 / 237$ & 1 & & $21 / 149$ & 1 & \\
\hline
\end{tabular}

$\mathrm{HPV}$, human papillomavirus; RR, relative risk; CI, confidence interval.

risk HPV infection is related to an increased risk of developing precancerous lesion or invasive carcinomas [20]. The persistence of high-risk HPV infection for 1 or 2 years strongly predicts a diagnosis of $\mathrm{CIN} 2+$ in the subsequent years [21,22]. Furthermore, high HPV viral load was significantly associated with lymphovascular space invasion and deep stromal invasion in cervical cancer [23].

In the analysis of type-specific prognoses, HPV 58 resulted in high rates of cytological progression at 6 and 12 months, compared with other high-risk types (RR, 3.30; 95\% CI, 2.04 to 5.30 ; $\mathrm{p}<0.001$ and RR, 5.03; 95\% CI, 2.56 to $9.91 ; \mathrm{p}<0.001$ ). The reason for the high prevalence of HPV 58 and cervical carcinogenesis in East Asia is not fully understood, but may result from differences in the oncogenic potentials among the high-risk HPV types. Also, the oncogenicity of viral variants and host genetic backgrounds may play roles in the distinct geographical distribution of HPV 58. A multi-step process involving various host factors is needed for development of tumor cells from an HPV-infected epithelium [24]. The prevalence of HPV 58 increases significantly according to the severity of cervical neoplasia, and its variants are epidemiologically associated with a higher oncogenic risk [11]. Four featured variant lineages of HPV 58 (sublineage A1) showing a regional preponderance in Asia, and associated with a higher risk for cervical neoplasia, were recently reported $[13,25]$. Therefore, the detection of HPV 58 in addition to HPVs 16 and 18 in cytologically abnormal women may provide an early indicator of cervical precancerous lesion and invasive cancers in East Asia. In addition, it may be helpful in predicting the effects of the next generation vaccines for other high-risk HPVs (non-HPV 16/18) [26].

This study was the first Korean HPV cohort study and provided a relatively large-scale assessment of the distribution of HPV types in South Korea. The results showed the impact of HPV genotypes on the natural course of cervical cytology over the course of 1 year. The evaluation of this cohort is ongoing and we will report follow-up data from the longitudinal study.

\section{Conclusion}

Overall, HPV genotypes numbered in the 50s were frequent in Korean women with ASC-US and LSIL. In particular, HPV 58 was the most common high-risk HPV other than HPV 16/18 and greatly contributed to cytological progression in women with ASC-US/LSIL. This result may help us to understand the HPV genotype distribution and provide additional information on the association of HPV types other than HPV 16/18 with the prognosis of abnormal cytology. 


\section{Conflicts of Interest}

Conflict of interest relevant to this article was not reported.

\section{Author Details}

${ }^{1}$ Department of Obstetrics and Gynecology, Cheil General Hospital and Women's Healthcare Center, Dankook University College of Medicine, Seoul, ${ }^{2}$ Division of AIDS, Center for Immunology and Pathology, National Institute of Health, Korea Centers for Disease Control and Prevention, Cheongwon, ${ }^{3}$ Department of Obstetrics and Gynecology, Keimyung University Dongsan Medical Center, Daegu, ${ }^{4}$ Department of Obstetrics and Gynecology, Chonnam National University Medical School, Gwangju, 5Department of Pathology, Cheil
General Hospital and Women's Healthcare Center, Dankook University College of Medicine, Seoul, ${ }^{6}$ Department of Obstetrics and Gynecology, Inje University Busan Paik Hospital, Inje University College of Medicine, Busan, ${ }^{7}$ Department of Preventive Medicine, College of Medicine, The Catholic University of Korea, Seoul, ${ }^{8}$ Department of Obstetrics and Gynecology, Seoul St. Mary's Hospital, College of Medicine, The Catholic University of Korea, Seoul, Korea

\section{Acknowledgments}

This study was supported by a fund of the Chronic Infectious Disease Cohort Study (4800-4859-304-260, 2013-E5100502) from the Korea Centers for Disease Control and Prevention.

\section{References}

1. de Villiers EM, Fauquet C, Broker TR, Bernard HU, zur Hausen H. Classification of papillomaviruses. Virology. 2004;324:17-27.

2. Ferlay J, Shin HR, Bray F, Forman D, Mathers C, Parkin DM. Estimates of worldwide burden of cancer in 2008: GLOBOCAN 2008. Int J Cancer. 2010;127:2893-917.

3. Jung KW, Won YJ, Kong HJ, Oh CM, Cho H, Lee DH, et al. Cancer statistics in Korea: incidence, mortality, survival, and prevalence in 2012. Cancer Res Treat. 2015;47:127-41.

4. Jung KW, Won YJ, Oh CM, Kong HJ, Cho H, Lee DH, et al. Prediction of cancer incidence and mortality in Korea, 2015. Cancer Res Treat. 2015;47:142-8.

5. Seol HJ, Ki KD, Lee JM. Epidemiologic characteristics of cervical cancer in Korean women. J Gynecol Oncol. 2014;25:70-4.

6. Smith JS, Lindsay L, Hoots B, Keys J, Franceschi S, Winer R, et al. Human papillomavirus type distribution in invasive cervical cancer and high-grade cervical lesions: a meta-analysis update. Int J Cancer. 2007;121:621-32.

7. Saslow D, Solomon D, Lawson HW, Killackey M, Kulasingam SL, Cain J, et al. American Cancer Society, American Society for Colposcopy and Cervical Pathology, and American Society for Clinical Pathology screening guidelines for the prevention and early detection of cervical cancer. CA Cancer J Clin. 2012;62: 147-72.

8. Munoz N, Bosch FX, Castellsague X, Diaz M, de Sanjose S, Hammouda D, et al. Against which human papillomavirus types shall we vaccinate and screen? The international perspective. Int J Cancer. 2004;111:278-85.

9. Lee WC, Lee SY, Koo YJ, Kim TJ, Hur SY, Hong SR, et al. Establishment of a Korea HPV cohort study. J Gynecol Oncol. 2013;24:59-65.
10. Solomon D, Davey D, Kurman R, Moriarty A, O'Connor D, Prey M, et al. The 2001 Bethesda System: terminology for reporting results of cervical cytology. JAMA. 2002;287:2114-9.

11. Chan PK, Lam CW, Cheung TH, Li WW, Lo KW, Chan MY, et al. Association of human papillomavirus type 58 variant with the risk of cervical cancer. J Natl Cancer Inst. 2002;94: 1249-53.

12. Hong D, Ye F, Chen H, Lu W, Cheng Q, Hu Y, et al. Distribution of human papillomavirus genotypes in the patients with cervical carcinoma and its precursors in Zhejiang Province, China. Int J Gynecol Cancer. 2008;18:104-9.

13. Li Y, Wang X, Ni T, Wang F, Lu W, Zhu J, et al. Human papillomavirus type 58 genome variations and RNA expression in cervical lesions. J Virol. 2013;87:9313-22.

14. An HJ, Cho NH, Lee SY, Kim IH, Lee C, Kim SJ, et al. Correlation of cervical carcinoma and precancerous lesions with human papillomavirus (HPV) genotypes detected with the HPV DNA chip microarray method. Cancer. 2003;97:1672-80.

15. Chan PK, Cheung TH, Li WH, Yu MY, Chan MY, Yim SF, et al. Attribution of human papillomavirus types to cervical intraepithelial neoplasia and invasive cancers in Southern China. Int J Cancer. 2012;131:692-705.

16. Ding DC, Hsu HC, Huang RL, Lai HC, Lin CY, Yu MH, et al. Type-specific distribution of HPV along the full spectrum of cervical carcinogenesis in Taiwan: an indication of viral oncogenic potential. Eur J Obstet Gynecol Reprod Biol. 2008;140: 245-51.

17. Plummer M, Schiffman M, Castle PE, Maucort-Boulch D, Wheeler CM; ALTS Group. A 2-year prospective study of human papillomavirus persistence among women with a 
cytological diagnosis of atypical squamous cells of undetermined significance or low-grade squamous intraepithelial lesion. J Infect Dis. 2007;195:1582-9.

18. Rodriguez AC, Schiffman M, Herrero R, Wacholder S, Hildesheim A, Castle PE, et al. Rapid clearance of human papillomavirus and implications for clinical focus on persistent infections. J Natl Cancer Inst. 2008;100:513-7.

19. Brown DR, Shew ML, Qadadri B, Neptune N, Vargas M, Tu $\mathrm{W}$, et al. A longitudinal study of genital human papillomavirus infection in a cohort of closely followed adolescent women. J Infect Dis. 2005;191:182-92.

20. Dalstein V, Riethmuller D, Pretet JL, Le Bail Carval K, Sautiere JL, Carbillet JP, et al. Persistence and load of high-risk HPV are predictors for development of high-grade cervical lesions: a longitudinal French cohort study. Int J Cancer. 2003;106: 396-403.

21. Castle PE, Rodriguez AC, Burk RD, Herrero R, Wacholder S, Alfaro $\mathrm{M}$, et al. Short term persistence of human papillomavirus and risk of cervical precancer and cancer: population based cohort study. BMJ. 2009;339:b2569.

22. Kjaer SK, Frederiksen K, Munk C, Iftner T. Long-term absolute risk of cervical intraepithelial neoplasia grade 3 or worse following human papillomavirus infection: role of persistence. J Natl Cancer Inst. 2010;102:1478-88.

23. Deng T, Feng Y, Zheng J, Huang Q, Liu J. Low initial human papillomavirus viral load may indicate worse prognosis in patients with cervical carcinoma treated with surgery. J Gynecol Oncol. 2015;26:111-7.

24. Chan PK. Human papillomavirus type 58: the unique role in cervical cancers in East Asia. Cell Biosci. 2012;2:17.

25. Chan PK, Luk AC, Park JS, Smith-McCune KK, Palefsky JM, Konno R, et al. Identification of human papillomavirus type 58 lineages and the distribution worldwide. J Infect Dis. 2011; 203:1565-73.

26. Joura EA, Giuliano AR, Iversen OE, Bouchard C, Mao C, Mehlsen J, et al. A 9-valent HPV vaccine against infection and intraepithelial neoplasia in women. N Engl J Med. 2015;372: 711-23. 